\title{
Intrauterine Growth Restriction Induced ECG Morphological Differences Measured in Adulthood
}

\author{
Nuria Ortigosa $^{1}$, Merida Rodriguez-Lopez ${ }^{2,3}$, Raquel Bailón ${ }^{4,5}$, Alvaro Sepulveda-Martinez ${ }^{2,6}$, \\ Eduard Gratacós ${ }^{2}$, Fátima Crispi ${ }^{2}$, Pablo Laguna ${ }^{4,5}$ \\ ${ }^{1}$ I.U. Matemática Pura y Aplicada, Universitat Politècnica de València, Spain \\ ${ }^{2}$ Fetal i+D Medicine Research Center,BCNatal-Barcelona Center for Maternal-Fetal and Neonatal \\ Medicine(Hospital Clínic and Hospital Sant Joan de Deu); IDIBAPS, University of Barcelona, \\ (CIBER-ER) \\ ${ }^{3}$ Pontificia Universidad Javeriana seccional Cali, Colombia \\ ${ }^{4}$ Aragón Institute of Engineering Research (I3A), University of Zaragoza, Spain \\ ${ }^{5}$ CIBER-BBN, Spain \\ ${ }^{6}$ Fetal Medicine Unit, Department of Obstetrics and Gynecology, Hospital Clinico Universidad de \\ Chile
}

\begin{abstract}
Numerous studies associate intrauterine growth restriction (IUGR) and premature birth with higher risk for cardiovascular diseases throughout adulthood. This study aims to describe changes at the surface ECG and observe if the results corroborate those correspondences found in previous studies of pre-adolescents who were born with low birthweight. 87 adults conformed the study population. There were 2 subgroups to study: term birth with adequate weight for gestational age (control group, 33 subjects), and those who had IUGR at birth (54 subjects). Once $Q R S$ and T-wave loops were obtained from the averaged vectorcardiogram, the angle between the depolarization and repolarization dominant vectors in the threedimensional space and the absolute and relative angles between the dominant vectors and the three orthogonal planes were studied. The angle between the dominant vectors of the QRS loop and the T-wave loop in the XY-plane (i.e. the frontal plane) showed statistically significant larger values for controls (13.49 \pm 13.65$)$ than for adults who were born with IUGR (9.26 18.47$)$. This narrowing can be hypothesised to be a biomarker for IUGR cardiovascular risk. The results are in accordance with those obtained for pre-adolescent subjects who were preterm, further supporting the findings.
\end{abstract}

\section{Introduction}

One of the main causes of low birth weight is intrauterine growth restriction (IUGR) [1], which is diagnosed when the fetus weight and mass are lower than normal with respect to the number of gestational weeks below the tenth percentile $[2,3]$.

Several references have studied and shown the correlation between the presence of low birth weight and cardiovascular pathologies in adulthood, such as coronary disease [4] or ischemic heart disease [5].

Previous studies have shown the heart morphology differences due to premature birth and IUGR on the ECG. These differences are reflected on several measurements obtained from the vectorcardiogram of pre-adolescent subjects $[6,7]$.

In this study we have analysed surface ECG from adult subjects who presented IUGR when were born with the aim of observing whether the morphological differences observed in pre-adolescent subjects still remain during adulthood.

\section{Materials}

The study population is conformed by 87 adult subjects whose 12-lead ECG has been acquired during 10 seconds at $1000 \mathrm{~Hz} .54$ adults suffered from IUGR inside the womb whereas 33 adults did not (control group). Table 1 shows the distribution of the cohort of subjects for which the study has been conducted. 
Table 1. Sex and age distribution of subjects included in the study.

\begin{tabular}{lccc}
\hline \hline Group & Male & Female & Average age (years) \\
\hline Control & 29 & 4 & 32.3 \\
IUGR & 22 & 32 & 28.4 \\
\hline \hline
\end{tabular}

\section{Methods}

\subsection{Dominant vectors of QRS and T loops}

First, ECG is delineated using wavelet transform, so that the onset and ofset of the individual waves (corresponding to QRS complexes and T waves) are detected [8]. Then, baseline wander is reduced. Next, the vectorcardiogram of length $K$ samples is obtained by means of the inverse Dower matrix [9], from which we can extract the corresponding depolarization and repolarization loops.

Both depolarization and repolarization loops are aligned with respect to the first available loops, respectively. For those alignments, the method presented in [10] is used, in order to also remove the influence of respiratory cycles.

Thus, loop alignment consists of three transforms: scaling (for loop expansion or compression), rotation (due to rotational changes of the heart), and shifts (to obtain time synchronization). Alignment can be described by equation 1 , where $Z$ and $Z_{R}$ are the $3 \times K$ and $3 \times K+2 \Delta$ matrices containing the loops and the aligned loops, respectively; $\alpha$ is the scaling parameter; $Q$ is the $3 \times 3$ rotation matrix, and $J_{\tau}$ is the shifting matrix corresponding to a $\tau$ shift. For a fully detailed explanation of the alignment process please see [10].

$$
Z=\alpha Q Z_{R} J_{\tau}
$$

Once alignment has been done, the average loop is obtained for each depolarization and repolarization set of loops. Finally, the dominant vectors of each average loop are obtained, which will indicate the dominant direction of the electric front during the QRS complexes and the T waves. Figures 1 and 2 show two examples of the obtained averaged loops for a control group subject and an IUGR group subject, respectively.

\subsection{Angle estimation}

Once we have obtained the dominant vectors for each average depolarization and repolarization loops, a set of angle measurements between them is obtained, such as the angle between the projections of these vectors on the frontal plane XY $\left(\theta_{R T-X Y}\right)$, the angles between the dominant vectors and each one of the three orthogonal planes

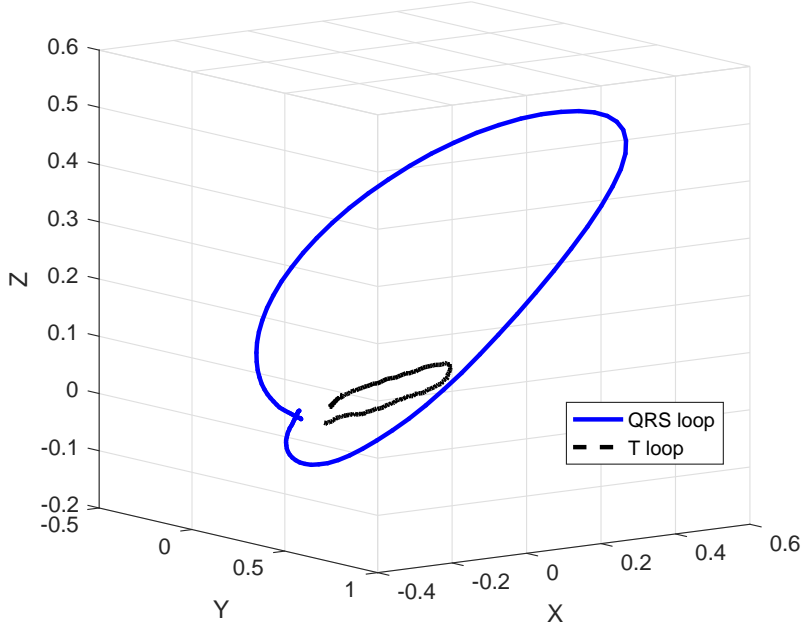

Figure 1. Depolarization (QRS) and repolarization (T) average loops of an adult subject of control group.

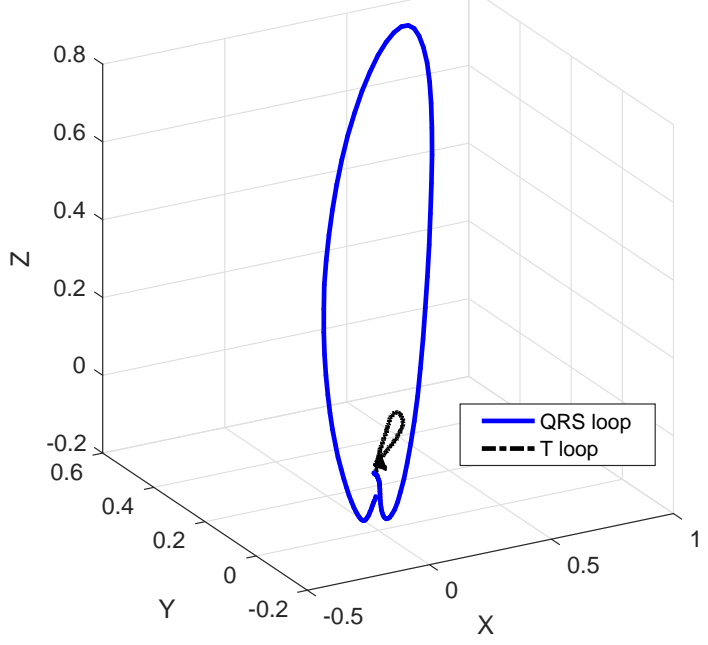

Figure 2. Depolarization (QRS) and repolarization (T) average loops of an adult subject of IUGR group.

$\left(\Phi_{R-P}\right.$ and $\Phi_{T-P}$, where $P$ is each one of the three planes $\mathrm{XZ}, \mathrm{ZY}$ and $\mathrm{YZ}$ ), and the differences between them.

\section{Results and discussion}

Table 2 shows the results of each one of the angles for the 87 adults included in the study. In addition, it also shows the p-values obtained by means of the Student's ttest. Statistical significant differences are considered if $\mathrm{p}$ value $<0.05$. 
Table 2. Obtained results for angles measured in degrees (mean \pm standard deviation) for control and IUGR groups. Statistically significant differences are indicated by * (if $\mathrm{p}$-value $<0.05$ ) or $* *$ (if $\mathrm{p}$-value $<0.01$ ).

\begin{tabular}{ccc}
\hline \hline Angle & Control group & IUGR group \\
\hline$\theta_{R T-X Y}$ & $13.49 \pm 13.65$ & $9.26 \pm 8.47^{* *}$ \\
$\Phi_{R-X Z}$ & $32.85 \pm 9.08$ & $35.68 \pm 6.12^{*}$ \\
$\Phi_{R-X Y}$ & $29.47 \pm 13.02$ & $22.07 \pm 11.80^{* *}$ \\
$\Phi_{R-Y Z}$ & $40.72 \pm 12.05$ & $44.17 \pm 9.81$ \\
$\Phi_{T-X Z}$ & $29.39 \pm 10.61$ & $33.15 \pm 10.67^{*}$ \\
$\Phi_{T-X Y}$ & $14.42 \pm 8.68$ & $14.48 \pm 9.41$ \\
$\Phi_{T-Y Z}$ & $54.83 \pm 9.52$ & $51.52 \pm 12.13$ \\
$\Phi_{R-X Z}-\Phi_{T-X Z}$ & $3.45 \pm 9.61$ & $2.54 \pm 8.43$ \\
$\Phi_{R-X Y}-\Phi_{T-X Y}$ & $15.05 \pm 14.14$ & $7.59 \pm 14.58^{* *}$ \\
$\Phi_{R-Y Z}-\Phi_{T-Y Z}$ & $-14.11 \pm 13.99$ & $-7.35 \pm 13.96^{* *}$ \\
\hline \hline
\end{tabular}

We can observe that the angle between the dominant vector of QRS complex and the dominant vector of T-wave projected on the frontal plane $\mathrm{XY}$ is significantly smaller (about $4^{\circ}$ ) for adults who had IUGR when they were born than for adults from the control group.

We can also observe that the angle between the dominant vector of the QRS loop and the XY-plane is also significantly smaller for IUGR group, whereas the angle between that vector and the XZ-plane is slightly larger for the control group.

It can be observed that the difference between the angles of the depolarization and repolarization vectors and XYand YZ- planes are larger (about $7^{\circ}$ ) for the control group as well.

On the other hand, when comparing these results (over an adult cohort) with the previously obtained over a cohort of pre-adolescents (presented in [6] and [7]), we can see that the frontal angle (i.e. the angle between the dominant vectors of QRS loops and T-waves projected on the frontal plane XY) was also statistically significantly larger for the control group. Thus, this angle could be a parameter of interest to be jointly studied with the rest of angles.

It would be necessary to do an extensive study on adults in order to obtain more details on these features of the surface electrocardiogram.

\section{Conclusions}

In this paper we have presented a study whose aim is to analyse the electrocardiogram differences of adults who were born with intrauterine growth restriction from those who were born with an appropriate weight.

We have analysed the angles between the dominant vectors of the loops corresponding to the QRS complexes (depolarization loops) and the T-waves (repolarization loops) from the vectorcardiogram.

We have found statistically significant differences (about $4^{\circ}$ degrees smaller values for the angle between the dominant vector of the QRS loop and the dominant vector of the T-wav loop in the frontal plane), which would define cardiovascular risk markers in adults who were born with IUGR.

\section{Acknowledgements}

N. Ortigosa acknowledges the support from Generalitat Valenciana under grant Prometeo/2017/102 and from Spanish MINECO under grant MTM2016-76647-P.

R. Bailón and P. Laguna acknowledge the support from Spanish MINECO and FEDER under grant DPI201675458-R, from Aragon Government and European Social Fund through BSICoS reference group (T39-17R), and from CIBER-BBN through Instituto de Salud Carlos III (Spain) and FEDER.

This project has been funded with support of the Erasmus + Programme of the European Union (Framework Agreement number: 2013-0040). This publication [communication] reflects the views only of the author, and the Commission cannot be held responsible for any use, which may be made of the information contained therein. Additionally, the research leading to these results has received funding form "la Caixa" Foundation under grant agreement LCF/PR/GN14/10270005, the Instituto de Salud Carlos III (INT16/00168, PI15/00130, PI14/00226, PI17/00675) integrados en el Plan Nacional de I+D+I y cofinanciados por el ISCIII-Subdirección General de Evaluación y el Fondo Europeo de Desarrollo Regional (FEDER) "Una manera de hacer Europa", Cerebra Foundation for the Brain Injured Child (Carmarthen, Wales, UK) and AGAUR 2017 SGR grant $n^{\circ} 1531$.

\section{References}

[1] Diderholm B. Perinatal energy metabolism with reference to iugr and sga: studies in pregnant women and newborn infants. Indian J Med Res 2009;130:612-617.

[2] Committee on Practice Bulletins-Gynecology, American College of Obstetricians and Gynecologists, Washington, USA. Intrauterine growth restriction. Clinical management guidelines for obstetrician-gynecologists. American College of Obstetricians and Gynecologists. Int $\mathrm{J}$ Gynaecol Obstet 2001;72(1):85-96.

[3] Figueras F, Gratacos E. Update on the diagnosis and classification of fetal growth restriction and proposal of a stage-based management protocol. Fetal Diagn Ther 2014; 36(2):86-98. 
[4] Barker D. The fetal and infant origins of adult disease. BMJ 1990;301(6761):1111.

[5] Ueda P, Cnattingius S, Stephansson O, Ingelsson E, Ludvigsson J, Bonamy A. Cerebrovascular and ischemic heart disease in young adults born preterm: a population-based swedish cohort study. Eur J Epidemiol 2014;29(4):253-60.

[6] Ortigosa N, Crispi F, Bailón R, Rodriguez-Lopez M, Gratacós E, Savari S, Sitges M, Bijnens B, Laguna P. Heart morphology differences induced by intrauterine growth restriction and premature birth measured on the ECG in preadolescents. Computing in Cardiology 2015;42:797-800.

[7] Ortigosa N, Rodriguez-Lopez M, Bailon R, Sarvari S, Sitges M, Gratacos E, Bijnens B, Crispi F, Laguna P. Heart morphology differences induced by intrauterine growth restriction and preterm birth measured on the ECG at preadolescent age. Journal of Electrocardiology 2016;49(3):401409.

[8] Martínez J, Almeida R, Olmos S, Rocha A, Laguna P. A
Wavelet-Based ECG Delineator: Evaluation on Standard Databases. IEEE Trans Biomed Eng 2004;51(4):570-581.

[9] Edenbrandt L, Pahlm O. Vectorcardiogram synthetized from a 12-lead ECG: Superiority of the inverse Dower matrix. J Electrocardiol 1988;21:361-367.

[10] Sörnmo L. Vectorcardiographic loop alignment and morphologic beat-to-beat. IEEE Trans Biomed Eng 1998; 45:1401-1413.

Address for correspondence:

Nuria Ortigosa

I.U. Matemática Pura y Aplicada,

Universitat Politècnica de València

Camino de Vera s/n, Edif. 8E, acceso F.

46022 Valencia (Spain)

nuorar@upvnet.upv.es 